\title{
Bowel cancer and previous mesh surgery
}

\author{
Sapna Ahuja • Oliver Chappatte - Michael Thomas • \\ Alistair Cook
}

Received: 27 October 2009 / Accepted: 24 January 2010 / Published online: 27 March 2010

(C) Springer-Verlag 2010

\begin{abstract}
We report two cases of large bowel cancer adjacent to mesh following previous abdominal sacrocolpopexy. As far as we are aware, there have been no previous reports of bowel cancer associated with mesh either in the form of a rectal erosion or mesorectal migration. In both cases, the mesh was part of the surgical field when operating for the large bowel cancer and had to be completely removed as it was intimately entangled with the surgical specimen to be removed. When carrying out procedures using mesh, it is important that we keep in mind that mesh surgery, especially for prolapse procedures, has been used for a relatively short duration of time, and there may still be unknown long-term complications associated with their usage. It is unlikely that the mesh is a causative agent in the above cases.
\end{abstract}

Keywords Bowel cancer $\cdot$ Mesh surgery $\cdot$ Abdominal sacrocolpopexy $\cdot$ Mesh complications $\cdot$ Mesh migration

\section{Case series: two cases of bowel cancer associated with mesh migration}

We report two cases of large bowel cancer adjacent to mesh following previous abdominal sacrocolpopexy. One of the cases was associated with rectal mesh erosion. In both cases, the mesh was part of the surgical field when

There is no falsification and fabrication of data, plagiarism, unethical experimentation and authorship disputes.

S. Ahuja $\cdot$ O. Chappatte $(\bowtie) \cdot$ M. Thomas $\cdot$ A. Cook

Obstetrics and Gynaecology,

Histopathology and Colorectal Surgery, Pembury Hospital,

Maidstone and Tunbridge Wells NHS Trust,

Kent, UK

e-mail: o.chappatte@nhs.net operating for the large bowel cancer and had to be completely removed as it was intimately entangled with the surgical specimen to be removed. As far as we are aware, there have been no previous reports of bowel cancer associated with mesh either in the form of a rectal erosion or mesorectal migration.

\section{Case 1}

A 53-year-old woman presented with a history of a small amount of rectal bleeding. She had had a vaginal hysterectomy 5 years ago followed by an abdominal sacrocolpopexy using mersilene mesh (type III mesh) 4.5 years ago.

General, abdominal, rectal examinations and proctoscopy were unremarkable. Sigmoidoscopy showed mesh eroding into the rectum. Subsequent colonoscopy confirmed this and also showed a malignant looking polyp in the rectosigmoid region, adjacent to the mesh. A biopsy of the polyp confirmed this to be an adenocarcinoma. Staging by computed tomography scan and magnetic resonance imaging (MRI) reported a T2 N0 M0 lesion.

At laparotomy, a radical anterior resection with total mesorectal resection and low colorectal anastomosis were carried out using staple guns. There was no suggestion of metastatic disease or extension. A densely fibrosed mesh was found stuck to the anterior surface of the sacrum passing straight into the rectum (Fig. 1). It was not attached to the vaginal vault which itself was well supported with no evidence of prolapse. The mesh was excised in its entirety with the specimen en bloc, taking a wide margin at its insertion into the sacrum. A bilateral salpingo-oophorectomy was performed at the same time.

Histology confirmed features of a P T1 N0 M0/Dukes' stage A cancer of moderate differentiation. There was no 




Fig. 1 Mesh eroding through the rectum

suggestion of extramural nodules or vascular invasion, and there was no evidence of tumour implantation within the mesh. Adjuvant radiotherapy and chemotherapy were not thought to be necessary.

The woman made an uneventful postoperative recovery and was followed up with tumour marker levels, clinically and radiologically. There was no evidence of local or metastatic spread, and there was no vault prolapse at 2 years postoperatively.

\section{Case 2}

A 73-year-old lady presented with symptoms of incomplete defecation and frequently opening her bowels three to four times per day. She had noticed blood and mucus on the paper following the first evacuation every day, but not in the pan or in stool. She also complained of a sensation of prolapse of the bowel and a suprapubic dull ache all the time limiting physical activity significantly.

She had recently undergone investigations for faecal soiling including anal ultrasound, anorectal physiology, pelvic MRI and proctoscopy which had confirmed a moderate cystocele and large rectocele which was shown to empty completely. The vaginal vault was well supported.

Mrs. SS had undergone an abdominal hysterectomy for fibroids in 1977, an anterior colporrhaphy in 1986, abdominal Burch colposuspension and posterior colpoperineorrhaphy in 1987 and a laparoscopic sacrocolpopexy and cystoscopy in 2003. During the sacrocolpopexy, polypropylene mesh (type I mesh) had been used to attach the vaginal vault to the sacral promontory after dissection of bowel adhesions from the sacral promontory. Following this, Mrs. SS had undergone a tension-free vaginal tape (TVT) for stress incontinence in 2005. All her operations had been carried out at different hospitals.

At colonoscopy in 1999 and 2003, dysplastic tubulovillous adenomas had been removed from the rectum and sigmoid colon. This was followed by a normal colonoscopy in 2004.
In view of the symptoms and previous history, a flexible sigmoidoscopy carried out in September 2007 reported an ulcerating cancer of the upper rectum $8-10 \mathrm{~cm}$ from the anal verge.

A radical low anterior resection, with total mesenteric excision, with low stapled anastomosis at $3 \mathrm{~cm}$ was performed in October 2007. At surgery, there was a large amount of mesh and mesh staples noted from previous surgery. The main specimen contained tumour and adjacent mesh which had become incorporated in the mesorectum. The mesh was also fixed to the sacrum by staples which had to be avulsed from the bone to release the mesh. The mesh was also excised from the vaginal vault. A part of the ileum was also excised due to mesh adherence causing an internal hernia, and a side-to-side stapled anastomosis was performed. A defunctioning transverse loop colostomy was also performed, and an omental pedicle was brought down to separate the low colorectal anastomosis from the vaginal vault closure to minimise the chance of rectovaginal fistulation.

A formal histology reported P T3 N0 M0/Dukes' B cancer with no extramural nodules and no vascular invasion and no invasion of the mesh. Figures 2 and 3 show histological images from the mesh fragment and vaginal vault in case 2 . Multi-disciplinary team review opinion was that there was no need for adjuvant chemotherapy.

A colonoscopy carried out through the stoma 4 months postoperatively demonstrated a mild stricture, which was dilated and the stoma closed.

\section{Discussion}

Colorectal cancer is the third most common cancer after breast and lung cancer, and it is the second most common cause of death from cancer in the UK after lung cancer [1-5]. Abdominal sacrocolpopexy is an operation carried out for vaginal vault prolapse where a natural or synthetic graft is used to suspend the vaginal vault to the sacrum. Where synthetic mesh is used as a graft, this can result in a vaginal mesh erosion in about $3.4-16 \%$ of cases [6, 7]. Rectal mesh erosions have been reported following mesh surgery for gynaecological and surgical disorders [8,9].

As far as we are aware, there have been no previous reports of bowel cancer associated with mesh either in the form of a rectal erosion or mesorectal migration. The bowel cancer in both cases is unlikely to be caused by the mesh, but chronic irritation by the mesh may be a contributing factor. There has been one case of vaginal leiomyosarcoma in the field of a prior TVT procedure using a Bard Duraderm allograft which is an acellular freeze-dried human dermal graft aseptically processed from donated human skin supplied by US tissue banks [10].

Several reports [11-13] have documented the carcinogenic potential of implanted foreign bodies including 
Fig. 2 High power images of mesh fragment, that on the right with polarised light demonstrating birefringence
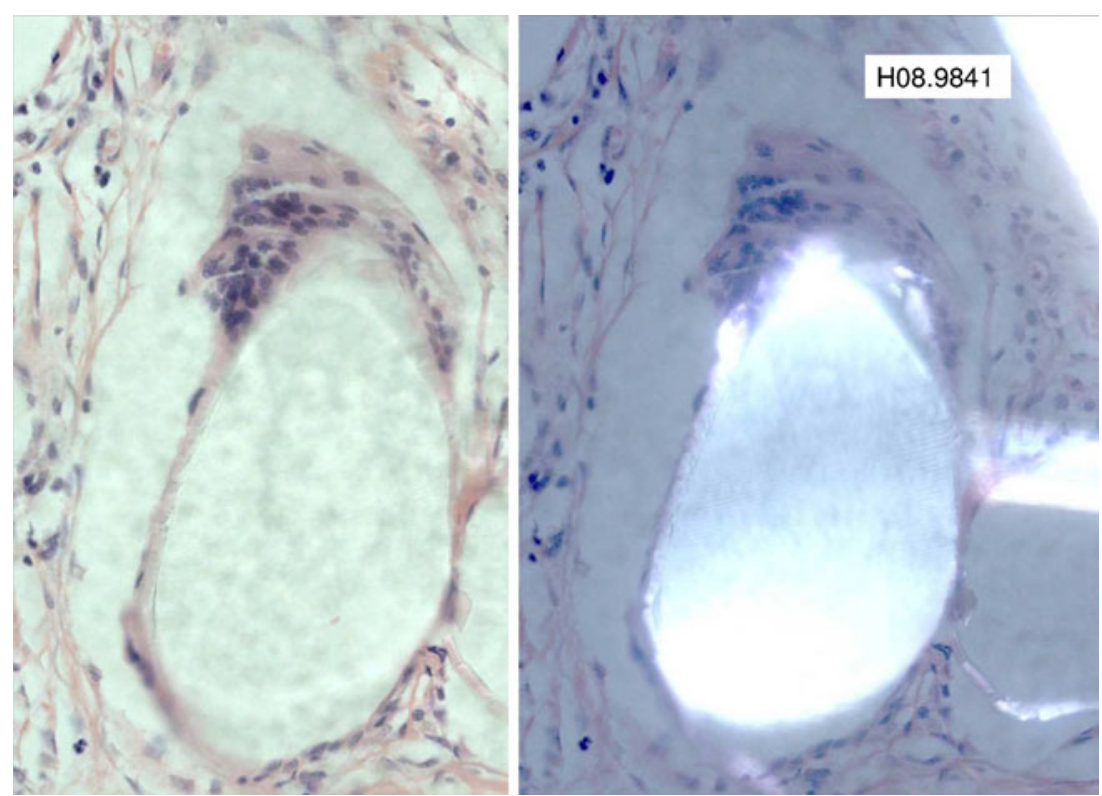

Dacron grafts, shrapnel, bullets, steel plates, bone wax and retained surgical sponges in humans. The implantation of a foreign body into tissues results in an inflammatory response. The foreign body reaction is transformed into a chronic reaction, the main participants of which are macrophages and giant cells. The long-term presence of a foreign body in a tissue in some cases results in emergence of sarcomas in its immediate vicinity [12]. Oppenheimer et al. [13] described results of subcutaneous implantation into rats of 16 types of plastic materials in the form of smooth continuous plates of equal shape and size. The frequency of tumour emergence in response to implantation of these materials varied within a rather broad interval. Elimination of contaminants did not decrease the tumorigenicity of the material.

In case 1 , there was no family history of cancer and no predisposing factors for bowel cancer. Case 2, however, had a family history of cancer and indeed had dysplastic polyps removed from the large bowel in 1999 and 2003. She had a normal colonoscopy in 2004 followed by the diagnosis of rectal cancer in 2007. The tumour clearly arose quite quickly in this case.

Bowel cancer is a fairly common cancer with around 100 new cases of colorectal cancer being diagnosed each day in the UK. Around two thirds are found in the colon and one third in the rectum [1-4].

Synthetic meshes are increasingly being used in the management of pelvic organ prolapse and stress incontinence. The use of suburethral slings for stress incontinence and the use of mesh for sacrocolpopexy are well documented. The Cochrane review on surgery for pelvic organ prolapse concluded that sacrocolpopexy is superior to the sacrospinous colpopexy in terms of preventing recurrent vault prolapse [14]. The success rate claimed for this
Fig. 3 Low power images of a section of tissue from the vaginal vault in case 2 , stained with $H \& E$, showing type 1 polypropylene mesh eliciting a fibrous tissue response with giant cells. The image on the right shows the birefringent nature of the mesh
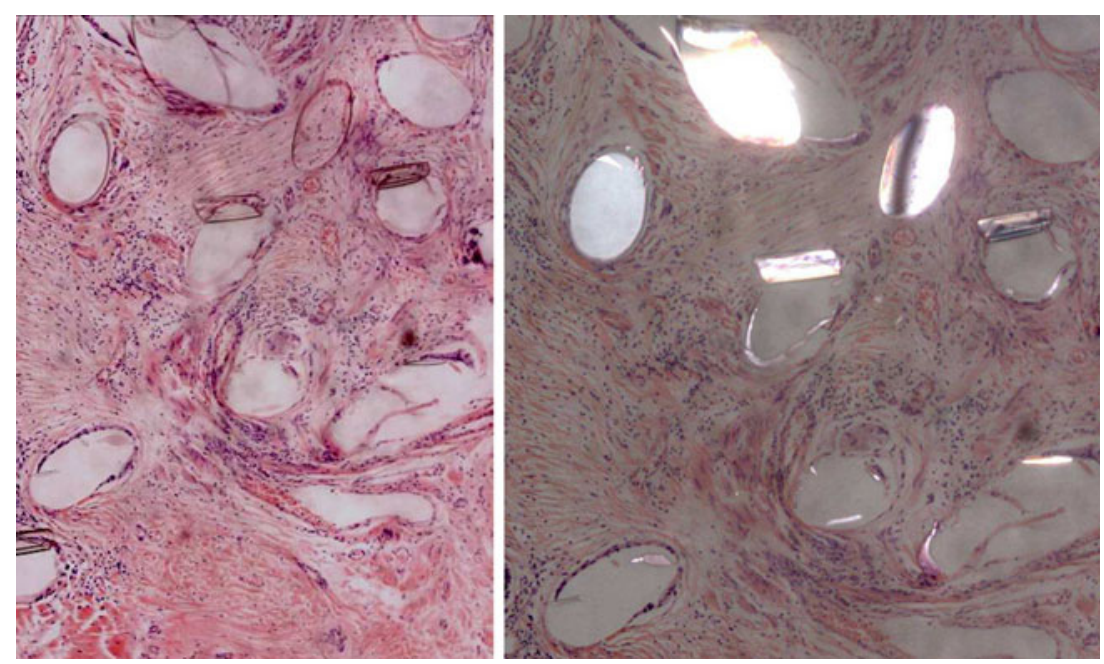
procedure is quoted as $84-99 \%[15,16]$. The aim of using mesh is to reduce the recurrence of prolapse following surgery and increase the longevity of repair.

Reported complications include mesh erosions and haemorrhage from the presacral vessels. Mesh erosion occurs in $4-16 \%$ of cases, typically occurring between 4 and 24 months after surgery [6,7] and is commonly a vaginal erosion. Overall, mesh-related complications are usually well below 6\% and, apart from erosions, also include sinus-tract formation, abscess, adhesions and obstructive ileus [14-17]. We did not come across any papers reporting cancers or tumours as a complication of mesh.

Mesh can be effective by replacing supportive tissue, reinforcement of inadequate tissue and generation of new tissue. Polypropylene mesh used in case 2 is a type I mesh which is macroporous with a pore size of $>75 \mu \mathrm{m}$. Macrophages are able to infiltrate the mesh, and the large pore size also allows the surrounding tissue and fibroblasts to incorporate the mesh, which is desired to generate a good support. It might however prove a problem if the bowel or the ureters are close, and adhesions might develop.

Elmer et al. [18] evaluated the histological inflammatory response to polypropylene transvaginal mesh and reported that this mesh induced a mild but persistent foreign body reaction. Histologically, postoperatively, there was an increase in the macrophage and mast cell counts but no significant change in the cell counts related to the infectious cell response or collagen density and the elastin area fraction at the mesh-tissue interface. Mersilene mesh used in case 1 is a type III mesh, which is macroporous with multifilament components. Although it theoretically admits bacteria, it does not admit macrophages, so infections may develop [19]. The use of mersilene mesh for sacral colpopexy is supported with an objective cure rate of $91 \%$ and complication rate of $15 \%$ [20]. Mesh responses also vary depending on site and route of implantation, host age and sex. Although all meshes seem to induce a host reaction of chronic inflammation with foreign body giant cells, lymphocytes and T-cells, it has been demonstrated that multifilament mesh may provoke more fibrosis as well as acute inflammation. In both our cases, there was extensive fibrosis with mesh eroding the rectum in case 1 and incorporating the mesorectum in case 2 .

Migration of mesh after hernia repair has been reported as primarily mechanical and secondarily due to erosion of surrounding tissue. Primary mechanical migrations are displacements of the mesh into adjoining anatomical spaces along paths of least resistance due to inadequate fixation or external displacing forces. In case 2 , the mesh was detached from the vaginal vault and densely incorporated in the mesorectum, and this was probably primary mechanical migration. Secondary migrations are slow and gradual movements through trans-anatomical planes. They are thought to be secondary to foreign body reaction induced erosion and are dependant on the nature and fixation of the mesh [21]. In case 1, the rectal erosion probably followed this mechanism.

\section{Conclusion}

Though there is emerging evidence that mesh surgery for prolapse is very effective and reduces the incidence of recurrence, most of this evidence is in the form of case series, and good randomised controlled trials are lacking. However, there are complications associated with mesh surgery. The mesh is a foreign body; long-term evidence is lacking and, hence, patients should be appropriately counselled.

Conflict of interest There is no conflict of interest.

Author's contributions The patients in the study were treated by Mr. Cook/Mr. Chappatte, and pathology was done by Dr. Thomas. Miss. Ahuja did the literature search, and the paper was written by all the authors.

\section{References}

1. Office for National Statistics (2004) Cancer statistics registrations: registrations of cancer diagnosed in 2004, England. Office for National Statistics, UK

2. Welsh Cancer Intelligence and Surveillance Unit (2007) Cancer incidence in Wales. Brunel House, Cardiff

3. ISD Online (2007) Cancer incidence and mortality data

4. Northern Ireland Cancer Registry (2007) Cancer statistics

5. Toms JR (2004) Cancer stats monograph 2004. Cancer Research UK, London

6. Kohli N, Walsh PM, Roat TW, Karram MM (1998) Mesh erosion after abdominal sacrocolpopexy. Obstet Gynecol 92(6):999-1004

7. Brizzolara S, Pillai-Allen A (2003) Risk of mesh erosion with sacral colpopexy and concurrent hysterectomy. Obstet Gynecol 102(2):306-310

8. Kenton KS, Woods MP, Brubaker L (2002) Uncomplicated erosion of polytetrafluoroethylene grafts into the rectum. Am J Obstet Gynecol 187(1):233-234

9. Hurtado EA, Bailey HR, Reeves KO (2007) Rectal erosion of synthetic mesh used in posterior colporrhaphy requiring surgical removal. Int Urogynecol J Pelvic Floor Dysfunct 18(12):1499 1501

10. Moller K, Mathes GL Jr, Fowler W Jr (2004) Primary leiomyosarcoma of the vagina: a case report involving a TVT allograft. Gynecol Oncol 94(3):840-842

11. Ben-Izhak O, Vlodavsky E, Ofer A, Engel A, Nitecky S, Hoffman A (1999) Epithelioid angiosarcoma associated with a Dacron vascular graft. Am J Surg Pathol 23(11):1418-1422

12. Moizhess TG (2008) Carcinogenesis induced by foreign bodies. Biochemistry (Mosc) 73(7):763-775, Review 
13. Oppenheimer BS, Oppenheimer ET, Danishevsky I, Stout AP, Eirich FR (1955) Further studies of polymers as carcinogenic agents in animals. Cancer Res 15:333-340

14. Maher C, Baessler K, Glazener CM, et al (2004) Surgical management of pelvic organ prolapse in women. Cochrane Database Syst Rev CD004014

15. Timmons MC, Addison WA, Addison SB, Cavenar MG (1992) Abdominal sacral colpopexy in 163 women with posthysterectomy vaginal vault prolapse and enterocele. Evolution of operative techniques. J Reprod Med 37(4):323-327

16. Culligan PJ, Murphy M, Blackwell L, Hammons G, Graham C, Heit MH (2002) Long-term success of abdominal sacral colpopexy using synthetic mesh. Am J Obstet Gynecol 187(6):14731480, discussion 1481
17. Nygaard IE, McCreery R, Brubaker L, Connolly A, Cundiff G, Weber AM, Zyczynski H (2004) Pelvic floor disorders network. Abdominal sacrocolpopexy: a comprehensive review. Obstet Gynecol 104(4):805-823

18. Elmer C, Blomgren B, Falconer C, Zhang A, Altman D (2009) Histological inflammatory response to transvaginal polypropylene mesh for pelvic reconstructive surgery. J Urol 181(3):1189-1195

19. Amid PK (1997) Classification of biomaterials and their related complication in abdominal wall hernia surgery. Hernia 1:15-21

20. Limb J, Wood K, Weinberger M, Miyazaki F, Aboseif S (2005) Sacral colpopexy using mersilene mesh in the treatment of vaginal vault prolapse. World J Urol 23(1):55-60

21. Agrawal A, Avill A (2006) Mesh migration following repair of inguinal hernia: a case report and review of literature. Hernia 10:79-82 